\title{
Dense bubble traffic in microfluidic loops: Selection rules and clogging
}

\author{
Virginie Hourtane,${ }^{1}$ Hugues Bodiguel,,${ }^{1, *}$ and Annie Colin ${ }^{2, \dagger}$ \\ ${ }^{1}$ Université Bordeaux, CNRS, Solvay, LOF, UMR 5258, 178 avenue Schweitzer, 33608 Pessac, France \\ ${ }^{2}$ ESPCI, SIMM UMR 7615, 11 rue Vauquelin, 75005 Paris, France \\ (Received 9 August 2015; revised manuscript received 10 January 2016; published 15 March 2016)
}

\begin{abstract}
We study the repartition of monodisperse bubbles at the inlet node of an asymmetric microfluidic loop for low to high bubble densities. In large loops, we evidence a new regime. Contrary to the classical belief, we point out that bubbles are directed not towards the arm having the higher total flow rate but towards the arm with the higher water flow rate at low and moderate relative gas flow rates. At higher rates, they enter the longer arm when they reach close packing in the shorter arm. In small loops, we evidence a clogging regime at high relative gas flow rates. Collisions between bubbles coming from the two arms at the outlet clog the longer arm. We propose a comprehensive analysis allowing us to explain these results.
\end{abstract}

DOI: 10.1103/PhysRevE.93.032607

\section{INTRODUCTION}

Foams are mixtures of gas and liquids we encounter everyday. In the last decades many applications dealing with the use of foams in soils have emerged. Surfactant foam technology has been suggested to achieve better mobility control in porous media and to improve removal efficiencies of several remediation processes. It has been investigated for removal of hydrophobic organic compounds [1] and heavy metals [2] from contaminated soils. This application is very promising, as there are many expected advantages of injecting a foam rather than a single-phase fluid in the polluted soil. It allows a large reduction of the volume of liquid needed for a given injection volume, while maintaining a very good compatibility with the surfactants already used for soil remediation. Foams may also be used as a substitute for polymer drive in the alkaline/surfactant/polymer (ASP) enhanced-oil-recovery (EOR) process [3]. In both EOR and soil remediation a foam flows through a porous material with a complex geometry. Understanding how water/gas systems behave and perform in porous media is critical to the effective application of foams. How foam flows in porous media is not always directly linked to how foam behaves in bulk form (e.g., when in a bottle). The effective viscosity of the foam depends upon the interactions between the bubbles and the porous media. An essential prerequisite for the applications is the knowledge of bubble traffic in the network. This is not an easy task, as flows of discrete objects such as bubbles thus differ from the flow of a simple fluid. It may become heterogeneous, involve the creation of preferential paths, and, in some cases, be chaotic [4].

In the last decades, these issues have stimulated many works [4-13]. These studies focus on a simple geometry: a simple loop or a simple junction. They contribute to the global picture of the flow, at least in the dilute dispersion situation and in regimes where the viscous stresses are so small that bubbles do not break. When a droplet or a bubble reaches the inlet of a junction having two outlets, it flows into the arm having

\footnotetext{
*Hugues Bodiguel Univ. Grenoble Alpes, CNRS, Lab. LRP UMR5520, F-38000 Grenoble, France.

†annie.colin@espci.fr
}

the higher flow rate. As a consequence, at high dilutions, all the droplets or bubbles flow in the shorter arm of an asymmetric loop. Since the presence of droplets or bubbles in a channel induces some extra dissipation, it lowers the flow rates. Thus, for more concentrated flows, a repartition of droplets or bubbles is observed in the arms of the loop. The transition between these two regimes occurs when the hydrodynamic resistance of the shorter arm becomes comparable to that of the longer arm. Then, if droplets alternatively go into the two arms, the mean hydrodynamic resistances of the two arms remain approximately equal in the limit of weak relative fluctuations in the number of droplets. This leads to an equality rule for the total flow rates [9,14-17].

In this work, we propose a rationalized experimental approach, by considering a basic yet essential element, a single loop, where the flow is separated into two streams. We perform a comprehensive study as a function of the dilution and of the size of the loop. We show that the size of the loop is an important parameter. In the large-loop situation, i.e., when the two arms are at least six times longer than the bubble size, as shown in previous studies [4-13], we evidence a filtering and a transition regime. Strikingly and contrary to previous studies [9,14-17] in the repartition regime situation, we point out that the hydrodynamic resistances or the total flow rates are never equal in the two arms. We note that this point was suggested by Engl [6] but not evidenced experimentally. In this new regime where interactions between bubbles cannot be neglected, we show that for low and moderate bubble densities, the continuous phase flow rates are equal in the two arms. When the distance between the bubbles in the shorter arm becomes comparable to the size of the bubble, the selection rule changes. Bubbles go into the longer arm and the bubbles in the shorter arm do not deform.

In the small-loop situation, we show that the size of the loop is an important parameter, and we evidence that a clogging regime follows the repartition regime when the bubble density is increased in the small-loop situation. To our knowledge, such a clogging has not been reported yet in the literature. This paper is presented as follows. In Sec. II, we describe the experimental procedure in detail. Section III is devoted to the study of bubble transport in a straight channel. Section IV deals with the study of the repartition of bubbles in a large loop as a function of the relative gas flow rate. We propose a simple model which 
(a)

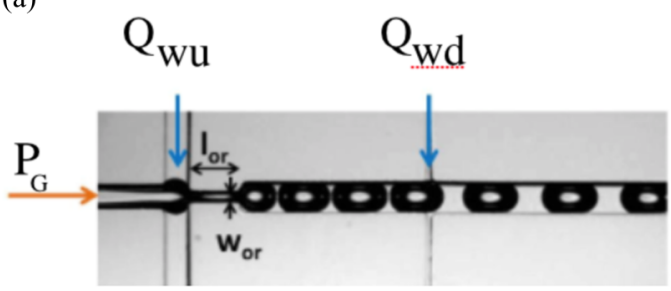

(b)

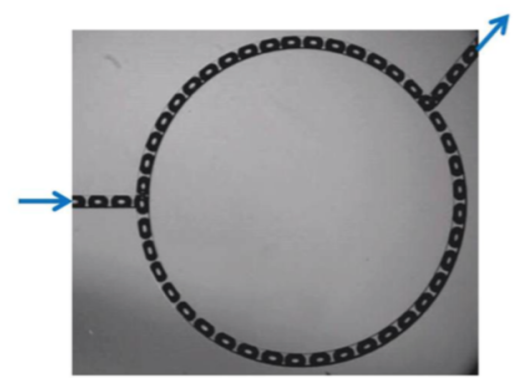

FIG. 1. Images of the device. (a) Flow focusing geometry: Bubbles are produced by a controlled water flow rate $Q_{w_{u}}$ and a gas pressure $P_{g}$. They are then diluted (or concentrated) with an additional (or removal) water flow rate $Q_{w_{d}}$. (b) Loop geometry: The bubble train then flows in a loop having two arms of different lengths, $L_{1}$ and $L_{2}$. In this picture, $L_{2}=2 L_{1}$. Channel widths are $100 \mu \mathrm{m}$ in both panels.

is validated by the experiments. In Sec. V, we move to the small-loop situation and and study the clogging regime.

\section{EXPERIMENTAL PROCEDURE}

We use polydimethyl-siloxane (PDMS) devices, made using standard microfabrication techniques. Bubbles are produced thanks to the flow-focusing geometry (see, for example, Ref. [18]); they are then diluted or concentrated by an additional water flow rate [see Fig. 1(a)]. The zone of study is located downstream and consists of a straight channel or of a loop having two arms of different lengths but of similar cross sections [see Fig. 1(b)]. The sections of the channels are square, of width $100 \mu \mathrm{m}$.

The air flow is imposed thanks to a pressure controller (Fluigent) at between 0 and $1400 \mathrm{mb}$ and syringe pumps control the aqueous solution flow rate. The aqueous solution contains $5 \%$, by weight, of a commercial dishwashing solution (Minimir), in order to avoid coalescence of the bubbles and to ensure total wetting. The surface tension $\gamma$ between the surfactant solution and the air is equal to $28 \mathrm{mN} / \mathrm{m}$. The gas pressure and the water flow rate at the level of the junction $Q_{w_{u}}$ are independently controlled and adjusted in order to form almost-monodisperse bubbles that are emitted at a constant rate $f_{0}$. The latter is systematically measured by performing a Fourier transform of the light intensity just after the constriction. Correlatively, the bubble volume $\Omega$ is determined by image analysis. This allows us to know the gas flow rate $Q_{g}$, given by $Q_{g}=f_{0} \Omega$. We adapt the water flow rate $Q_{w_{u}}$ in order to get a constant bubble volume, independently of the gas flow rate. Despite this procedure, the bubble volume varies slightly (up to 10\%) from one experimental condition to another.
An additional injection or removal of water downstream $Q_{w_{d}}$ dilutes or concentrates the system and increases or reduces the distance $\lambda$ between two successive bubbles while keeping their volume unchanged (see Fig. 1). The channel is thus filled with a periodic train of droplets moving at a constant velocity $V_{b}=\lambda f$ at a water flow rate $Q_{w}=Q_{w_{u}}+Q_{w_{d}}$, which is directed towards the loop or the straight channel. The relative gas flow rate (foam quality) is defined as $q=Q_{g} / Q_{T}$, where the total flow rate $Q_{T}$ is given by $Q_{T}=Q_{w}+Q_{g}$.

We record videos of the moving bubbles using a fast video camera at a typical acquisition rate of 500 frames/s and use image processing software (MATLAB) to determine the bubble frequency and velocity. The frequency measurement is achieved by determining the maximum of the temporal Fourier transform of a single pixel and the velocity by spatial correlations.

In order to characterize the pressure drop in a straight channel, we incorporate in the device two small channels that are directly connected to a pressure sensor. The pressure sensor is a differential analogic captor (Honeywell Ref. No. AXDXRR015PDAA3). It contains sensing elements that consist of four piezoresistors buried in the face of a thin, chemically etched silicon diaphragm. Our captor measures pressure in the range $0-15$ psi (i.e., $1.03421 \mathrm{~b}$ ) with a precision of $\pm 2 \mathrm{mb}$. The frequency of acquisition is equal to $2 \mathrm{~Hz}$. The small dimensions of the pressure sensor channels prevent bubbles from getting in. Pressure drop measurements were first validated with pure water; the data agree well with the Poiseuille law up to $10 \%$.

\section{BUBBLES IN STRAIGHT CHANNELS}

We consider bubbles flowing in a square or in a rectangular channel. We consider regimes where the viscous effects are much smaller than the surface tension effects (low capillary number) and where inertial effects are negligible compared to viscous effects (low Reynolds number).

\section{A. Bubble velocity}

Let us first focus on bubble velocities. For confined bubbles in square or rectangular geometries, this issue is not straightforward because the bubbles do not fill the entire section. The carrier fluid can flow through the corners of the section, either backward or forward in the reference frame of the bubbles.

Figure 2 displays the experimental bubble velocity as a function of the total flow rate $Q_{T}=Q_{w}+Q_{a}$ for two straight geometries. These experiments correspond to a range of capillary numbers $\mathrm{Ca}=\eta Q_{T} / \gamma S$ ( $S$ being the area of the cross section and $\eta$ the aqueous phase viscosity) between $10^{-4}$ and $10^{-2}$ and to a relative gas flow rate $q=Q_{g} / Q_{T}$ between 0.1 and 0.8 . We found that the bubble velocity $V_{b}$ varies linearly with the total flow rate, i.e., $V_{b}=\beta Q_{T} / S$. Interestingly, the value of $\beta$ does not depend on the bubble interdistance even in the concentrated regime where the wavelength of the bubble trains is of the order of the bubble size. $\beta$ seems to depend on the channel aspect ratio or on the bubble size, as $\beta=0.81$ in a square channel of width $w=100 \mu \mathrm{m}$ for bubbles of length $L_{b}=1.4 w$ and $\beta=0.93$ in a rectangular channel of cross section $w \times h=250 \times 300 \mu \mathrm{m}$ for bubbles of length $L_{b}=1.6 w$ (see Fig. 2). 

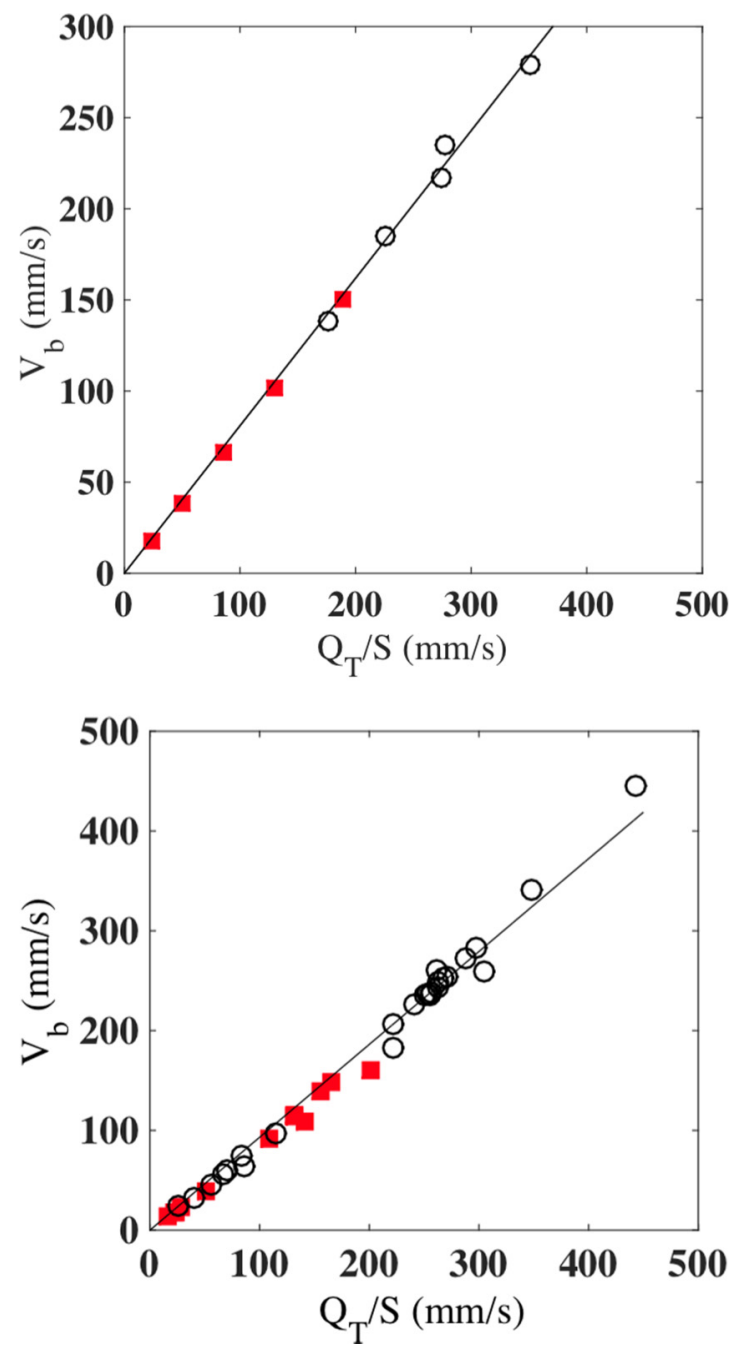

FIG. 2. Droplet velocity as a function of the total flow rate in a square channel of characteristic size $w=100 \mu \mathrm{m}$ (top) and in a rectangular channel of of width $w=250 \mu \mathrm{m}$ and height $h=300 \mu \mathrm{m}$ (bottom). Open black circles correspond to the concentrated regime $\left(\lambda>2 L_{b}\right)$, and filled red squares to the dilute one $\left(\lambda<2 L_{b}\right)$. The relative size of the bubbles is equal to $L_{b} / w=1.4$ (top) and $L_{b} / w=$ 1.6 (bottom). The line corresponds to a linear fit associated with a $\beta$ value equal to 0.81 (top) and to 0.93 (bottom).

\section{B. Drop in pressure}

The presence of a bubble in a channel strongly modifies the velocity profile of the carrier fluid around it and thus the pressure gradient. It has been proposed [19] that the pressure drop along a channel of length $L$ and of width $w$, containing a density of $n$ bubbles per unit of length, could be modeled as

$$
\Delta P=\frac{L \gamma}{w^{2}}\left[a\left(1-n L_{b}\right) \mathrm{Ca}+b n L_{b} \mathrm{Ca}+c n w \mathrm{Ca}^{2 / 3}\right],
$$

where $L_{b}$ is the bubble length, and $a, b$, and $c$ are numerical parameters of geometrical constraints. The first term, linear with respect to the velocity, represents the contribution of the carrier fluid flow between the bubbles. The parameter $a$ depends only on the channel aspect ratio and equals 28.5 for square channels. The second term is proportional to the number of bubbles and is due to the liquid flow through the gutters [19] but can also incorporate the consequence of the perturbation of Poiseuille flow upstream and downstream a droplet. The third is also proportional to the number of bubbles and corresponds to the drop in pressure induced by the viscous dissipation in the transitional region between the hemispherical end caps of the bubbles and the thin films. It was first calculated by Bretherton for circular tubes [20] and then by Wong et al. for rectangular channels [21]. It is worth noting that in the presence of a layer of surfactant with a high interfacial dilatational modulus, the value of $c$ differs from the stress-free interface case and an extra pressure drop scaling, as $\mathrm{Ca}^{1 / 3}$ has to be considered [22]. Let us emphasize that, at least in the presence of surfactants, the parameters $b$ and $c$ need to be experimentally characterized.

We take advantage of the knowledge of the first term in Eq. (1) in order to experimentally determine the extra pressure drop due to a single bubble $\Delta P_{\text {bubble, defined as }}$ $\Delta P_{\text {bubble }}=\Delta P / n L-\gamma\left(1-n L_{B}\right) / n w^{2}$. Figure 3 displays the results obtained from direct pressure drop measurement
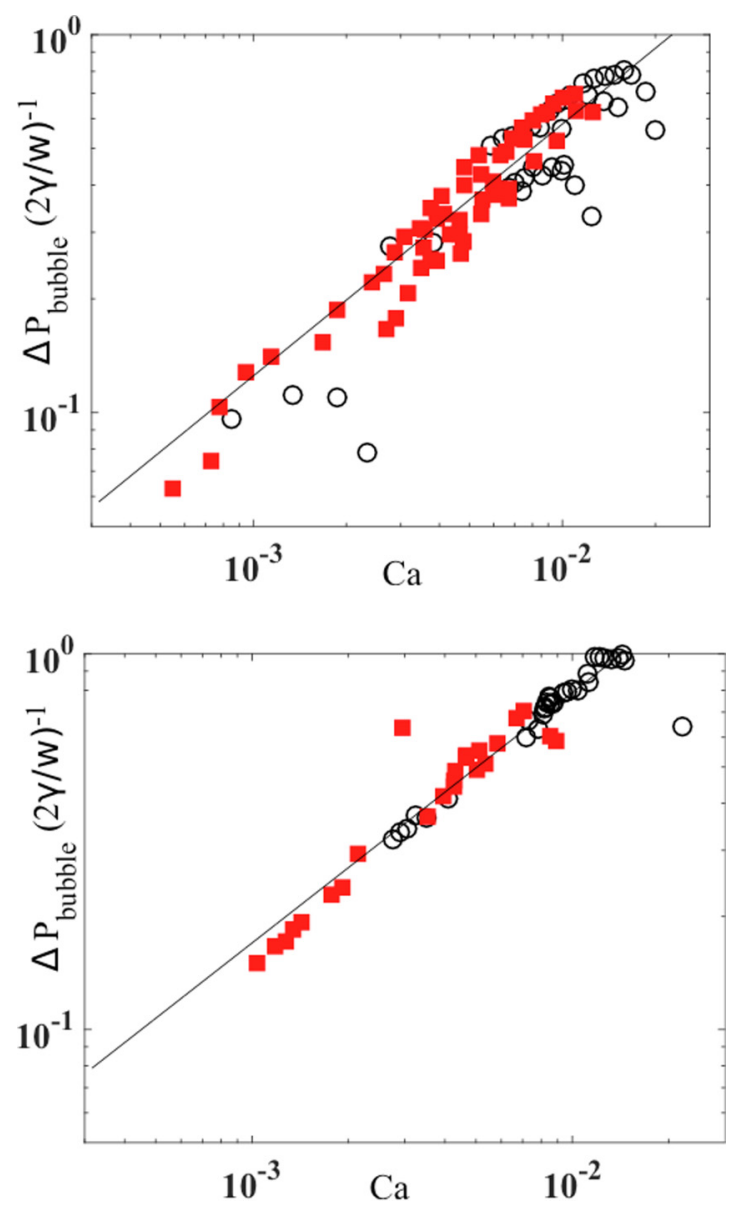

FIG. 3. Pressure drop due to a single bubble $\Delta P_{\text {bubble }}$ (see text), normalized by $2 \gamma / w$, plotted as a function of the capillary number for various relative gas flow rates. The line represents the best fit to the data using a power law of exponent $2 / 3$. It corresponds to the model (see text) with $a=28, b=0$, and $c=25$ (top) or $c=34$ (bottom). Experiments were performed in a square channel of $100 \mu \mathrm{m}$ (top) and in a rectangular channel of $250 \times 300 \mu \mathrm{m}$ (bottom). The relative bubble size is $L_{b} / w=1.4$ (top) and $L_{b} / w=1.6$ (bottom). Open black circles correspond to the concentrated regime $\left(\lambda>2 L_{b}\right)$, and filled red squares to the dilute one $\left(\lambda<2 L_{b}\right)$. 
in two channels. In both cases, the data exhibit a clear scaling in $\mathrm{Ca}^{2 / 3}$. This implies that, in the range of $\mathrm{Ca}$ studied and for the rather small bubbles investigated, the second term in Eq. (1) could be neglected, similarly to the work by Parthiban and Khan [14,23]. The best fits to the data lead to $c \simeq 28$ for the square channels and $c \simeq 34$ for the rectangular one (see Fig. 3). These values differ from the theoretical predictions of Wang et al. and from those determined experimentally [14,19]. This is not surprising, as this parameter depends upon the channel aspect ratio and on the nature and concentration of the surfactant [19].

Importantly for the following and as highlighted in Fig. 3, the extra pressure drop per bubble remains the same for concentrated bubble trains. This observation might be related to the fact that the main contribution to the pressure drop is the Bertherton-like term [third term in Eq. (1)]. In the following section where we consider only channels of square cross section, we thus use Eq. (3) to model the pressure drop, with $a=28.5, b=0$, and $c=25$.

\section{Discussion}

Let us now come back to the observation that the bubble velocity $V_{b}$ is linear with respect to the total flow rate and slightly less than unity. This is, at first sight, surprising since the pressure drop due to a bubble scales as $\mathrm{Ca}^{2 / 3}$, and one may thus expect some kind of nonlinear dependency of the relative velocity. Due to the small thickness of the wetting films, the flow of the carrier fluid in the vicinity of the bubble occurs principally through the gutters that exist in square or rectangular geometries. This flow rate $Q_{\text {gutter }}$ could be estimated from the pressure drop acting on the bubble $\Delta P_{\text {bubble }}$ and the hydrodynamic resistance in the gutters, so that $Q_{\text {gutter }} \simeq \Delta P_{\text {bubble }} w^{2} / \alpha^{\prime} \eta_{w}\left(L_{b}-w\right)$, where the numeric constant $\alpha^{\prime}$ has been computed using COMSOL [24] and equals approximately $1.8 \times 10^{4}$ [26].

The total flow $Q_{T}$ rate could be expressed in the mid cross section of a bubble and is given by $Q_{T}=\left(S-S_{\text {gutter }}\right) V_{b}+$ $Q_{\text {gutter }}$, where $S_{\text {gutter }}=w^{2}(1 / 4-\pi / 16)$ is the total area of the gutters. By combining the expression of the pressure drop, $\Delta P_{\text {bubble }}=c \mathrm{Ca}^{2 / 3} \gamma / w$, with the previous equation, we obtain the following expression for the coefficient $\beta=S V_{b} / Q_{T}$ :

$$
\beta=\frac{1}{1-S_{\text {gutter }} / S+A \mathrm{Ca}^{-1 / 3}},
$$

where the constant $A$ is given by $A=c w / \alpha^{\prime}\left(L_{b}-w\right)$. This analysis leads to a value of $\beta$ that should increase with increasing capillary number, with typical values of 0.98 for $\mathrm{Ca}=10^{-4}$ and 1.02 for $\mathrm{Ca}=10^{-3}$. Given the rather small range of velocities tested experimentally, it is thus not surprising to observe a roughly linear variation of the bubble velocity versus the total flow rate. We note, however, that the experimental values of $\beta$ are smaller than the prediction. This might indicate that in the above analysis, the flow through the gutters is a bit underestimated. Additional experiments at lower capillary numbers would be useful to gain some insight into this issue. In the following, we use the experimental and constant value of $\beta$ to model the flow in loops.

The most important conclusion of this section is the fact that for both the relative velocity of the bubbles and the pressure drop expression, the measured relations in the dilute regime are still valid in the concentrated one. Even for closely packed bubble trains, the pressure drop is still given by expression (1) and the bubble velocity by $V_{b}=\beta Q_{T} / S$.

\section{BUBBLES IN LARGE LOOPS}

We consider a loop with two arms of different lengths $L_{1}<$ $L_{2}$ but of the same cross section (see Fig. 1). We first study large loops, diameter $6.4 \mathrm{~mm}$, of square channels of width $w=100 \mu \mathrm{m}$. The bubble size is $L_{b} \simeq 1.4 \mathrm{w}$, so that for these large loops $L_{i} \gg L_{b}$.

As reported in the literature, we evidence two regimes: a filter regime at low relative gas flow rates and a repartition regime at higher rates (see Fig. 4). The transition between these two regimes strongly depends on the asymmetry between the two arms; it occurs at lower relative gas flow rates for $\Lambda=L_{2} / L_{1}=5$ than for $\Lambda=2$.

In order to describe the bubble traffic quantitatively, we extract from the videos the bubble velocities $V_{b_{i}}$ and frequencies $f_{i}$ in each of the two arms ( $i=1$ and 2 for the short and long arms, respectively). It is straightforward to deduce the gas flow rates as $Q_{g_{i}}=f_{i} \Omega$ from these measurements. As the bubble velocity is proportional to the total flow rate (see previous section), the flow rates in each arm are computed according to $Q_{T_{i}}=V_{b_{i}} S / \beta$. Let us note that as the two channels are the same size, bubbles will flow at the same velocity if the total flow rate is the same in the two arms. The knowledge of the $\beta$ value is thus not required to estimate the ratio between the two total flow rates. Figure 5 displays the evolution of the normalized total flow rate in each arm for two aspect radii, $\Lambda=L_{2} / L_{1}=2$ and $\Lambda=5$, as a function of the relative gas flow rate $q=Q_{g} / Q_{T}$. The flow rate in the short arm decreases with increasing relative gas flow rate in the filtration regime, reaches a minimum at the transition, and then increases (Fig. 6).
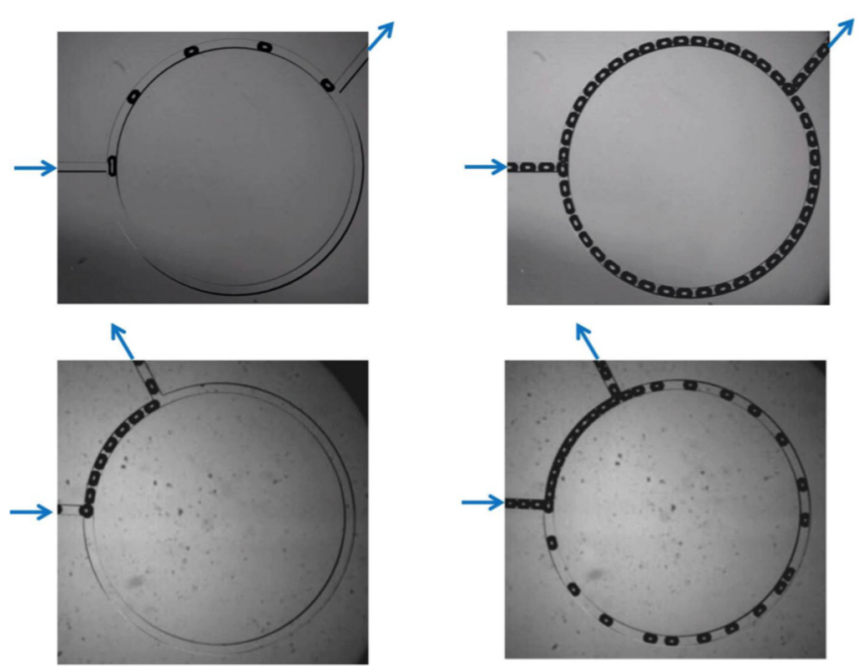

FIG. 4. Pictures of bubble traffic in the large loop. Top left: Filter regime in a loop where $\Lambda=L_{2} / L_{1}=2$. Top right: Repartition regime at a high relative gas flow rate in the same loop. Bottom left: Repartition regime in a loop where $\Lambda=5$. Bottom right: Repartition regime in a loop where $\Lambda=5$. Arrows indicate the flow direction. 


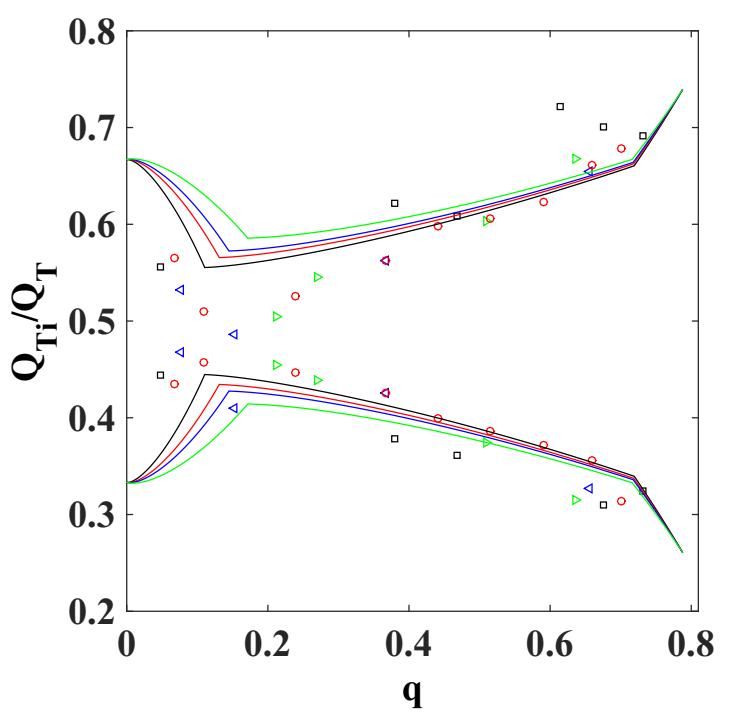

FIG. 5. Relative total flow rate $Q_{T_{i}} / Q_{T}$ for bubbles in the short and in the long arm as a function of the relative gas flow rate in large loops $\Lambda=2$. Values $>0.5$ are for the short arm, and lower ones for the long arm. Different symbols correspond to various gas flow rates, which are made nondimensional by defining $\mathrm{Ca}^{*}=\eta Q_{g} / \gamma S$. Black squares and black lines represent $\mathrm{Ca}^{*}=10^{-3}$; red circles and red lines, $\mathrm{Ca}^{*}=2 \times 10^{-3}$; blue leftward triangle and blue lines, $\mathrm{Ca}^{*}=$ $3 \times 10^{-3}$; and green rightward triangles and green lines, $\mathrm{Ca}^{*}=6 \times$ $10^{-3}$. Solid lines correspond to the model. From top to bottom the capillary number decreases and is, respectively, equal to $6 \times 10^{-3}$, $3 \times 10^{-3}, 2 \times 10^{-3}$, and $10^{-3}$. Note that contrary to the work of Engl et al. [25], the model does not correspond to a single curve in the parameter-plane relative total flow rate or relative gas flow rate. This is due to the nonlinearity of the pressure drop with respect to the capillary number.

To model the flow in the loop, we need to predict the evolution of four quantities: the wavelengths $\lambda_{1}$ and $\lambda_{2}$ and the total flow rates $Q_{T_{1}}$ and $Q_{T_{2}}$. Conservation equations for each phase together with the pressure drop balance $\Delta P_{1}=\Delta P_{2}$ provide three equations. To complete the set of equations, one thus needs an additional equation which accounts for the selection rule at the inlet junction. Path selection at the junction is governed by viscous forces. Since these are proportional to the carrier fluid velocity, one could expect that the bubble will move towards the arms where the total flow rate is the highest. Many works [9,14-17] have indeed argued that this selection rule applies. In the repartition regime, it implies that the flow rate difference between the arms fluctuates around 0 . Averaging over time, both flow rates should be about the same. However, the data displayed in Fig. 7 disagree with this picture, as the fractional flow rate in the shorter arm is significantly higher than 0.5 over the whole range of relative gas flow rates studied.

We thus proceed by analyzing the experimental features of the flow. As shown in Fig. 8, we found that the water flow rates $Q_{w_{i}}=Q_{T_{i}}-Q_{g_{i}}$ are roughly equal for low and moderate relative gas flow rates $(q \lesssim 0.5)$. This observation has been reported by Engl et al. [25]. However, it seems hard to provide a comprehensive interpretation. Careful examination of the videos reveals several features that might explain why the flow

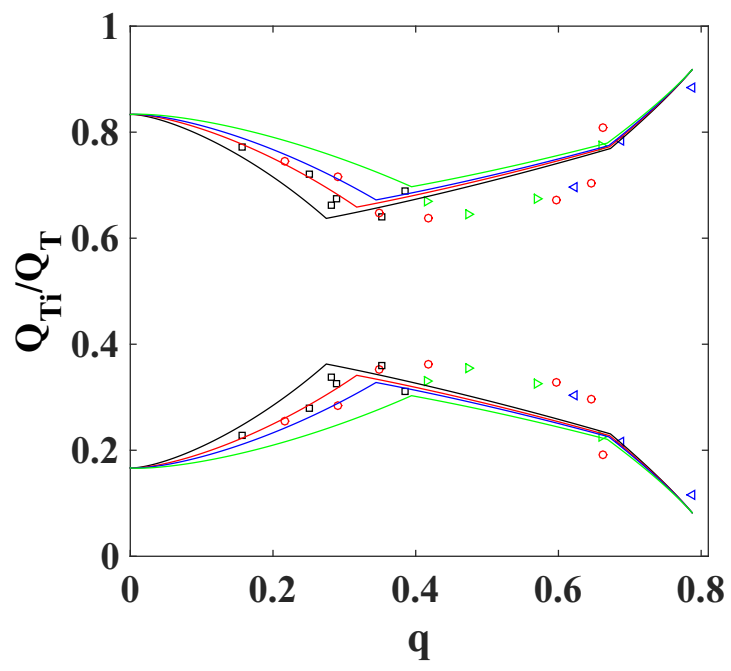

FIG. 6. Relative total flow rate $Q_{T_{i}} / Q_{T}$ for bubbles in the short and in the long arm as a function of the relative gas flow rate in large loops $\Lambda=5$. Same symbols as in Fig. 5 .

rates in both arms are not equal on average. Let us first stress that in these situations the bubbles do not touch but are quite close to each other. The wavelength at the transition between the filter and the repartition regime is between 2 and $3 L_{b}$ (see Fig. 9). These numbers are greater than unity but are not very large. They suggest that in the observed regime bubbles may interact. Bubbles remain at the inlet junction for a significant fraction of the temporal period between bubbles before moving to one of the arms. This creates some collisions between bubbles that may contribute to the path selection [11]. We also note that the total flow rate exhibits some fluctuations in time, associated with the bubble production. In the following,

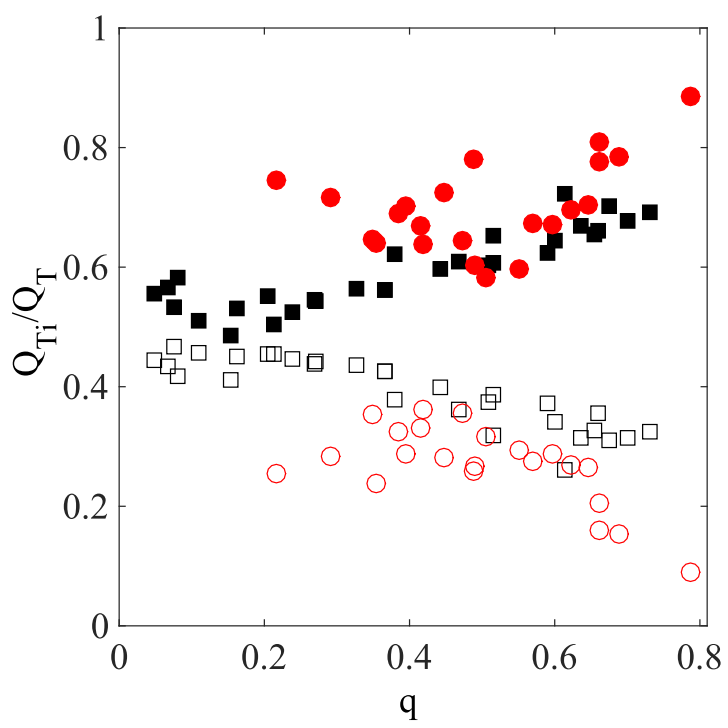

FIG. 7. Flow rate in the arms of the loop $Q_{T_{i}}$, normalized by the total flow rate $Q_{T}$, for two geometries as a function of the relative gas flow rate. Black squares correspond to a loop with $\Lambda=L_{2} / L_{1}=2$; red circles, to a loop with $\Lambda=5$. Open symbols correspond to data collected in the long arm. The two total flow rates are never equal. 


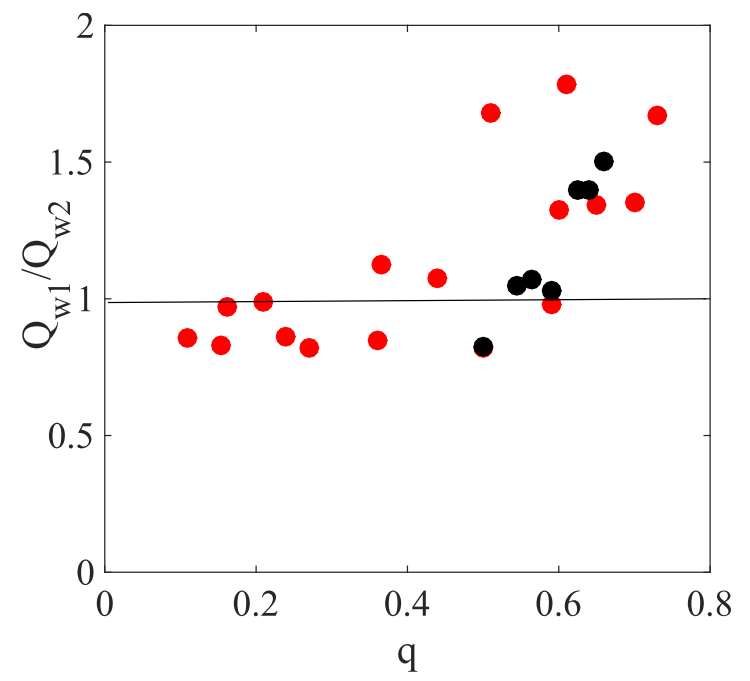

FIG. 8. Ratio of water flow rate in the short arm $Q_{w_{1}}$ to that in the long arm $Q_{w_{2}}$, as a function of the relative gas flow rate $q$. Water flow rates are deduced from the mean bubble velocity $V_{b_{i}}$ and frequency $f_{i}$ measurements, assuming that $Q_{w_{i}}=S V_{b_{i}} / \beta-\Omega f_{i}$. For $q<0.5$ the water flow rates are equal in the two arms. Red (gray) symbols correspond to $\Lambda=2$; black symbols, to $\Lambda=5$.

we use, in the repartition regime, the empiric selection rule at the inlet junction, that is, $Q_{w_{1}}=Q_{w_{2}}$.

For $q>0.6$, we observe that neither the total flow rates nor the water flow rates are equal in the two arms. Looking in more detail at the features of the flow, we note that these situations correspond to experiments where the spatial period in the shorter arm is approximately equal to the length of the bubble. This is highlighted in Fig. 9. In other words, at high relative gas flow rates, close packing is reached in the shorter arm, and bubbles go into the longer one even if the water flow rate is lower in the longer arm. In this concentrated regime, bubble collisions at the inlet are responsible for entering the longer arm, as the density in the shorter arm is too high. We note that this mechanism differs from the one proposed by Belloul et al. [11]. In their approach, limited to dilute trains of droplets, the authors also claim that collisions at the junction impact the selection rule at the junction, based on the observation that two colliding droplets are always directed towards different arms. In the present study, the role of collisions is slightly different, as they promote bubble traffic in the longer arm due to close packing in the shorter.

Knowing the selection rule at the junction, modeling the bubble traffic is straightforward. In all the regimes, mass and momentum conservation allows us to write

$$
\begin{aligned}
\Delta P_{1} & =\Delta P_{2}, \\
Q_{T} & =Q_{T_{1}}+Q_{T_{2}}, \\
Q_{g} & =Q_{g_{1}}+Q_{g_{2}} .
\end{aligned}
$$

This set of equations has to be complemented by a regimedependent selection rule. In the filtering regime occurring at low relative gas flow rates,

$$
Q_{g_{2}}=0
$$
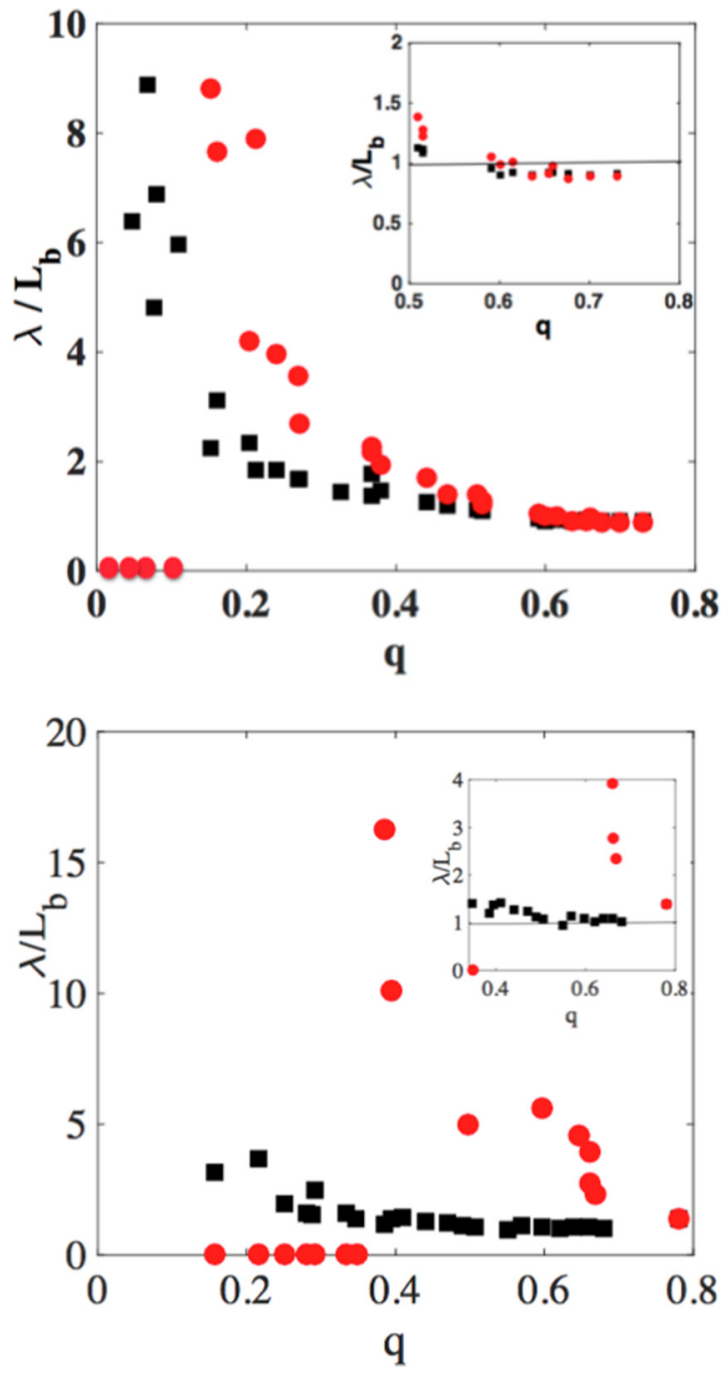

FIG. 9. Mean distance $\lambda_{i}$ between bubbles in the two arms (red squares, long arm; black squares, short arm) as a function of the relative gas flow rate $q$ in a loop where $\Lambda=2$ (top) or where $\Lambda=5$ (bottom). For high values of $q$, the mean distance between bubbles in the short arm is equal to the length of the bubble. In the filtering regime, the distance between bubbles is arbitrarily set to 0 in the long arm.

In the moderate repartition regime, we use the empiric condition that the liquid flow rates are the same in the two arms, i.e., $Q_{w_{1}}=Q_{w_{2}}$ or, equivalently,

$$
Q_{T_{1}}-Q_{g_{1}}=Q_{T_{2}}-Q_{g_{2}} .
$$

In the concentrated repartition regime, we state that the wavelength in the shorter arm is equal to the bubble size, $\lambda_{1}=$ $L_{b}$. Given the proportionality between the bubble velocity and the total flow rate, $V_{b}=\beta Q_{T} / S$, the above condition could be expressed as

$$
Q_{g_{1}}=Q_{T_{1}} / \alpha_{1}
$$

where $\alpha_{1}=S L_{b} / \beta \Omega$.

Using Eq. (1) with $b=0$ to model the pressure drops, these equations can be written using two dimensionless variables, the fraction of flow rate in the shorter arm $v_{1}=Q_{T_{1}} / Q_{T}$ and 

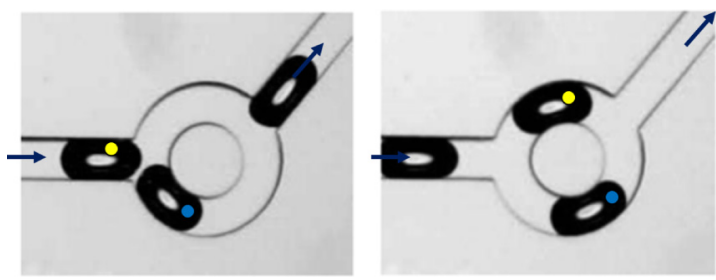

FIG. 10. Pictures of the flow in a small loop, in the repartition regime. $Q_{w}=1000 \mu \mathrm{l} / \mathrm{h}, Q_{g}=360 \mu \mathrm{l} / \mathrm{h}$. Successive pictures are separated by $2 \mathrm{~ms}$.

the normalized gas flow rate in the shorter arm $q_{1}=Q_{g_{1}} / Q_{T}$. We obtain the following equation, which holds for all three regimes:

$$
\frac{v_{1}-\alpha_{1} q_{1}+\alpha_{2} q_{1}\left(v_{1} \mathrm{Ca}\right)^{-1 / 3}}{1-v_{1}-\alpha_{1}\left(q-q_{1}\right)+\alpha_{2}\left(q-q_{1}\right)\left[\left(1-v_{1}\right) \mathrm{Ca}\right]^{-\frac{1}{3}}}=\Lambda,
$$

where the two parameters $\alpha_{1}=S L_{b} / \beta \Omega$ and $\alpha_{2}=S w c / \beta \Omega a$ are determined experimentally (see previous section) and are $\alpha_{1}=1.65, \alpha_{2}=0.735$. The above equation is completed by a selection rule, which depends on the regime. In the filtering regime, $q_{1}=q$; in the moderate repartition regime, liquid flow rate equality implies that $q_{1}=v_{1}+q / 2-1 / 2$; and in the concentrated repartition regime, $q_{1}=v_{1} / \alpha$.

Equation (7) is easily solved numerically, allowing us to predict $v_{1}$ as a function of $q$ and $\mathrm{Ca}$, together with the boundaries of the regimes. Due to the nonlinear term in $\mathrm{Ca}^{-1 / 3}$, it is not possible to provide analytical expressions for these. The results are shown in Fig. 5, together with the experimental data, for the three fixed $\mathrm{Ca}^{*}=q$ Ca tested experimentally.

Despite the scatter in the data (which might come from uncertainties and variations of the bubble size), the model captures semiquantitatively the measured flow rates, over the whole range of relative gas flow rates and for the two loops investigated. Let us recall that there is no adjustable parameter since the two parameters involved were determined independently. We could thus conclude that in large loops, mean-field arguments are sufficient to account for bubble traffic.

\section{BUBBLES IN SMALL LOOPS}

Let us now consider small loops, i.e., loops where the length of the short arm is a few times the bubble length. We have investigated loops of diameter $720 \mu \mathrm{m}$ with an aspect ratio of $\Lambda=2$ of $\Lambda=5$ and loops of diameter $2100 \mu \mathrm{m}$ with an aspect ratio of $\Lambda=2$.

\section{A. Experimental data for the loop of diameter $720 \mu \mathrm{m}$, with an aspect ratio of $\Lambda=2$}

Strikingly, for this small loop, the features of the flow totally differ from those for the large loops described in the previous section. At low and moderate relative gas flow rates, the filter and repartition regimes remains qualitatively similar. Figure 10 shows an example of the repartition regime. We note that due to the small lengths of the arms, there is no more well-defined steady-state repartition, but a periodic one. Upon increasing

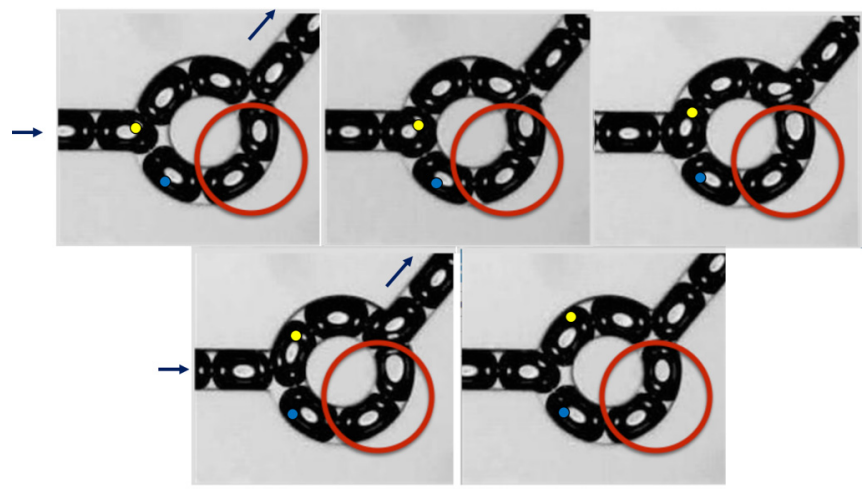

FIG. 11. Pictures of the flow in a small loop, in the clogging regime. Bubbles in the long arm are trapped. $Q_{w}=250 \mu \mathrm{l} / \mathrm{h}, Q_{g}=$ $100 \mu \mathrm{l} / \mathrm{h}$. Successive pictures are separated by $0.4 \mathrm{~ms}$.

the relative gas flow rate further, we evidence a new regime, referred to hereafter as the clogging regime, in which some bubbles are trapped in the long arm. This is shown in Figs. 11 and 12. This clogging is permanent: bubbles remain in the long arm for minutes, and the only possibility of remobilizing them is to increase the flow rate significantly (see Supplementary Material). Bubbles in the longer arm bear the action of bubbles exiting the shorter arm when arriving at the outlet junction. These collisions prevent them from exiting the loop. Due to these collisions, trapped bubbles move back and forth in the longer arm over distances which are of the order of a small fraction (typically a tenth) of the bubble length.

Let us note that the number of trapped bubbles may be one, two, or three (see Figs. 11 and 12). The empty space is filled with water. We note that clogging occurs for bubbles of length $184 \mu \mathrm{m}$ as well as for bubbles of length $165 \mu \mathrm{m}$ (see Figs. 12 and 13). Thus, the exact size of the bubbles is not a crucial parameter, and the clogging phenomenon may not be attributed to the fact that the arm length would be a multiple of the bubble length.

When the flow rate is high enough, for Ca greater than $4 \times 10^{-3}$, we observe that this clogging regime switches to
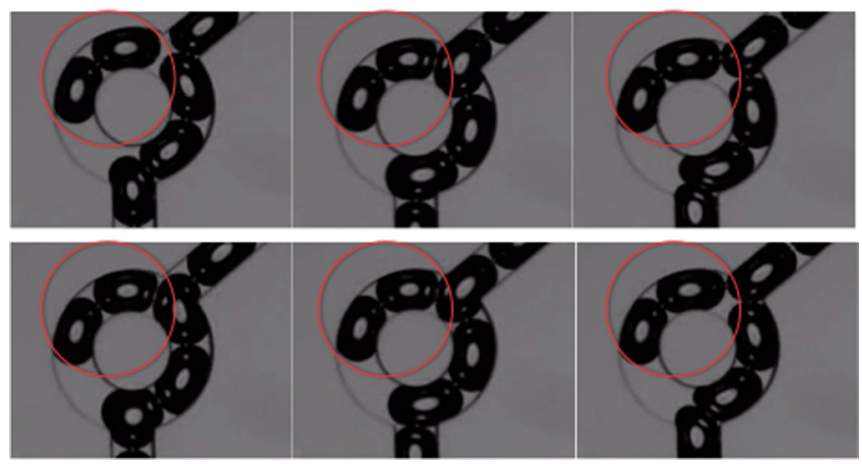

FIG. 12. Pictures of the flow in a small loop, in the clogging regime. Bubbles in the long arm are trapped. Note that only two bubbles are trapped and that the other part of the arm is filled with water. $Q_{w}=100 \mu \mathrm{l} / \mathrm{h}, Q_{g}=60 \mu \mathrm{l} / \mathrm{h}$. In this experiment, the bubble length is equal to $165 \mu \mathrm{m}$. Successive pictures are separated by 0.6 ms. 


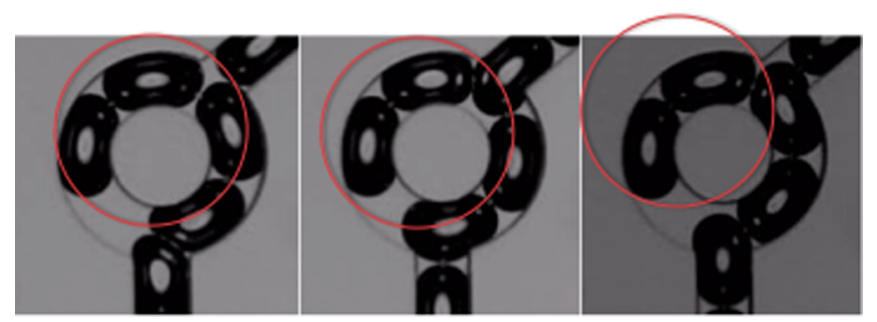

FIG. 13. Pictures of the flow in a small loop, in the clogging regime. Bubbles in the long arm are trapped. $Q_{w}=100 \mu \mathrm{l} / \mathrm{h}, Q_{g}=$ $60 \mu \mathrm{l} / \mathrm{h}$. In this experiment, the bubble length is $184 \mu \mathrm{m}$. Successive pictures are separated by $0.6 \mathrm{~ms}$.

a repartition regime, which is qualitatively different from the one at low and moderate relative gas flow rates: the collisions associated with the clogging regime remain but the bubbles deform to go through the outlet instead of moving back. This is illustrated in Fig. 14, which shows that two bubbles exit the loop at the same time.

Figure 15 summarizes these observations. The bubble frequency in the long arm is 0 at low relative gas flow rates, i.e., in the filtering regimes. It then increases upon entering the repartition regime before vanishing again in the clogging regime (except for the highest value of $\mathrm{Ca}^{*}=\mathrm{Ca} / q$ tested, i.e., $\left.6 \times 10^{-3}\right)$. The data indicate that the transition from the repartition regime to the clogging one occurs for $q$ between 0.5 and 0.7 and seems to increase.

\section{B. Experimental data for the loop of diameter $720 \mu \mathrm{m}$, with an aspect ratio of $\Lambda=5$}

Let us now discuss the role of the aspect ratio $\Lambda$. Figure 16 displays the observations. In this situation, we do not observe a repartition regime. For a given capillary number, at low relative gas flow rates, we first observe a filtering regime. The frequency $f$ of the bubbles in the long arm is equal to 1 . At higher relative gas flow rates, some bubbles enter the long arm but remain trapped and we again observe the clogging mechanism. As for $\Lambda=2$, this clogging regime switches to a repartition regime where the bubbles deform to go through the

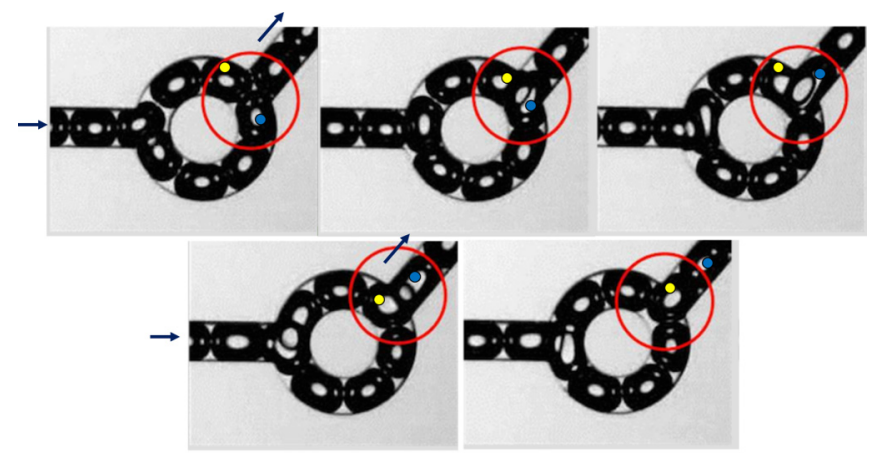

FIG. 14. Pictures of the flow in a small loop, in the repartition regime following the clogging regime. Bubbles deform to exit the loop. Successive pictures are separated by $0.5 \mathrm{~ms}$. $Q_{w}=125 \mu \mathrm{l} / \mathrm{h}$, $Q_{g}=600 \mu \mathrm{l} / \mathrm{h}$.

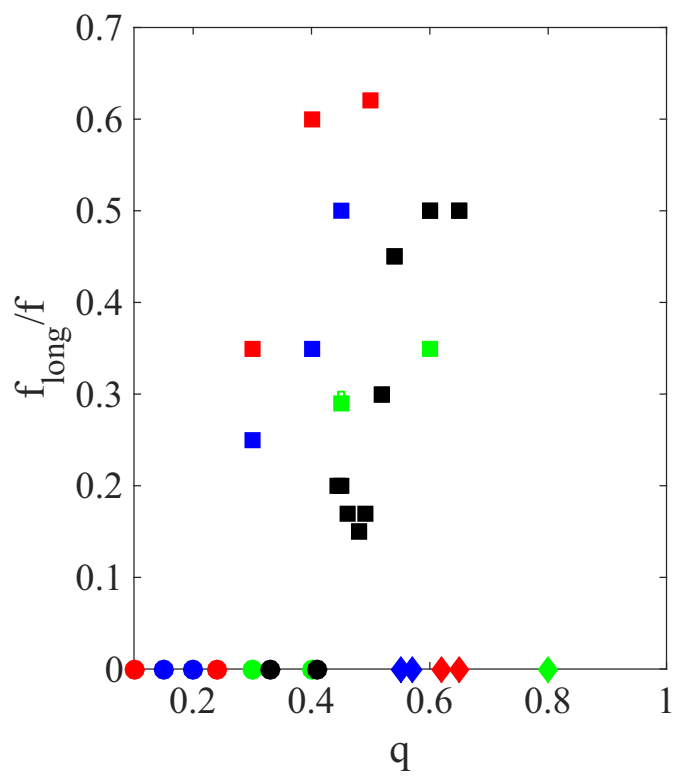

FIG. 15. Bubble rate in the long arm divided by the initial bubble frequency $\Lambda=2$. Circles correspond to the filtering regime; squares, to the repartition regime; and diamonds, to the clogging regime. Each color corresponds to a given gas flow rate, made nondimensional according to $\mathrm{Ca}^{*}=\eta Q_{g} / \gamma S$. Red symbols correspond to $\mathrm{Ca}^{*}=$ $10^{-3}$; blue symbols, to $\mathrm{Ca}^{*}=2 \times 10^{-3}$; green symbols, to $\mathrm{Ca}^{*}=3 \times$ $10^{-3}$; and black symbols, to $\mathrm{Ca}^{*}=6 \times 10^{-3}$. Note that the clogging regime disappears for high values of $\mathrm{Ca}^{*}$.

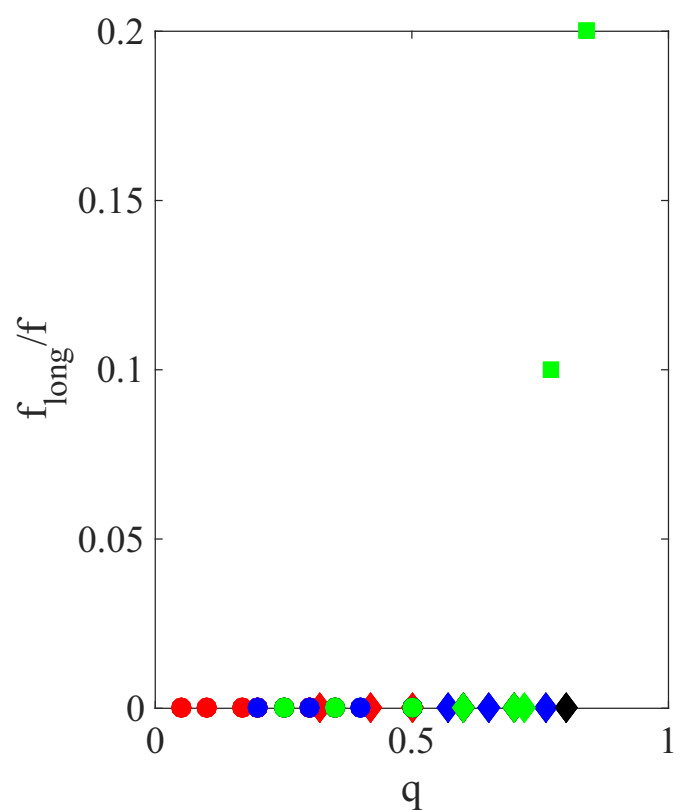

FIG. 16. Bubble rate in the long arm divided by the initial bubble frequency for $\Lambda=5$. Circles correspond to the filtering regime; squares, to the repartition regime; and diamonds, to the clogging regime. Each color corresponds to a given gas flow rate, made nondimensional according to $C a^{*}=\eta Q_{g} / \gamma S$. Red symbols correspond to $\mathrm{Ca}^{*}=10^{-3}$; blue symbols, to $\mathrm{Ca}^{*}=2 \times 10^{-3}$; green symbols, to $\mathrm{Ca}^{*}=3 \times 10^{-3}$; and black symbols, to $\mathrm{Ca}^{*}=6 \times 10^{-3}$. Note that the clogging regime disappears for high values of $\mathrm{Ca}^{*}$. 


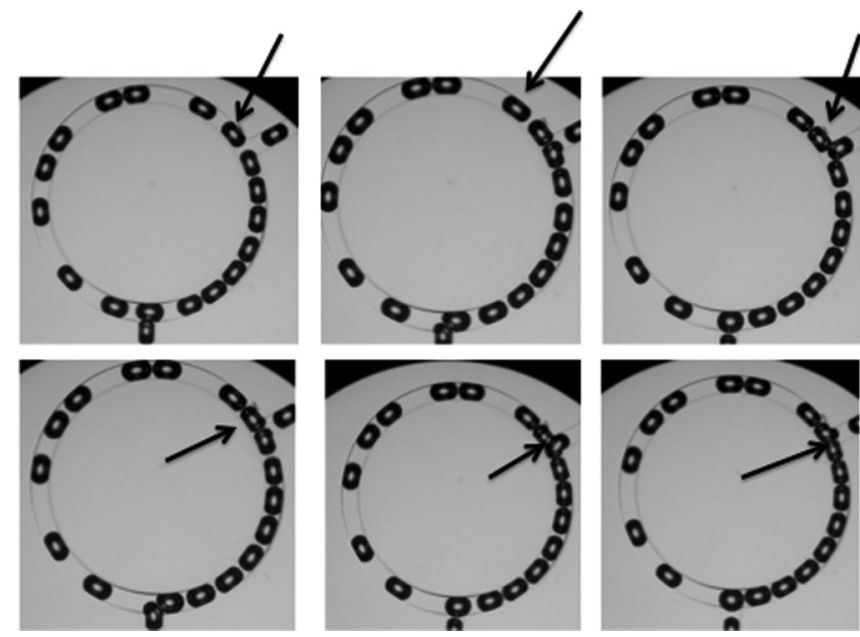

FIG. 17. Pictures of the flow in an intermediate loop, in the repartition regime. Bubbles in the long arm are dynamically blocked before leaving the loop. Successive pictures are separated by $0.5 \mathrm{~ms}$. $Q_{w}=200 \mu \mathrm{l} / \mathrm{h}, Q_{g}=600 \mu \mathrm{l} / \mathrm{h}$. Arrows indicate the same bubble.

outlet. The behavior is thus the same, but the clogging regime occurs for a higher foam quality when the flow rate is set.

\section{Experimental data for the loop of diameter $2100 \mu \mathrm{m}$, with an aspect ratio of $\Lambda=2$}

To obtain a better description of the mechanism in charge of the flow, we decided to conduct experiments on a loop with an intermediate diameter, $d=2100 \mu \mathrm{m}$. In this situation, we observe qualitatively the same behavior as in large loops. Clogging no longer occurs. However, as in the small-loop situation, there is no more well-defined steady-state repartition but, rather, a periodic one. The description of the flow does not follow the previous modeling and is beyond the scope of this paper. Figure 17 shows some pictures of the flow. We note that dynamic congestions occur at the outlet, leading to clogging and declogging of the long arm.

To conclude this experimental section, let us summarize the main results. These experiments point out a new regime in the repartition of bubbles at the junction: a clogging regime. This clogging regime occurs when the size of the long arm is close to the size of the bubbles.

\section{A crude model to explain clogging}

The clogging regime is rather puzzling. Examination of the videos indicates that it is related to congestion of the outlet of the loop and to bubble collisions. However, it is very specific to small loops, as we never observed it in large loops despite careful investigations at very high relative gas flow rates. In the following, we discuss the possible mechanism associated with this regime.

Let us analyze the pressure field in the loop. One could assume as a first approximation that the pressure gradient in the shorter arm should still be given by Eq. (1), as the flow remains unperturbed. Neglecting the first term since the clogging regimes occurs at high bubble densities, $\Delta P_{1} \simeq$ $c n L_{1} \mathrm{Ca}^{2 / 3} \gamma / w$. In the longer arm of the loop, the flow vanishes, on average, the clogging regimes. We have checked, by seeding the water with small tracer particles, that the water velocity between bubbles in this arm remains negligible compared to that in the shorter arm. Therefore, except in the vicinity of the last bubble, the pressure gradient in the longer arm is negligible. Thus, $\Delta P_{1}$ is balanced by a pressure drop localized on the last bubble of the longer arm, $\Delta P_{\text {clogging. }}$ This drop in pressure comes from the collision at the junction: the bubble suddenly moves back, which creates a counter-flow of water through the gutters along the bubble. This is the only possibility for $\Delta P_{\text {clogging }}$ to balance $\Delta P_{1}$.

The pressure drop associated with the counter-flow through the gutters can be estimated as $\Delta P_{\text {clogging }}=$ $\alpha\left(L_{b}-w\right) \eta_{w} Q_{\text {gutter }} / w^{4}$, where $Q_{\text {gutter }}$ is the flow rate in the gutters and $\alpha$ is a numeric constant. The value of $\alpha$ has been calculated using COMSOL and is about $1.1 \times 10^{5}$ [24]. The water flow rate $Q_{g}$ might be estimated as the frequency of the collision $f_{1}$ (i.e., the bubble rate in the shorter arm) times the displaced volume, which we assume to be a fraction $\delta$ of the bubble volume $\Omega$. Thus $Q_{\text {gutter }} \simeq \delta f_{1} \Omega=\delta Q_{g}$. Note that $\delta$ might be slightly dependent on the capillary number, as we observe that the amplitude of the back-and-forth movement decreases with increasing $\mathrm{Ca}$.

The above analysis provides a criterion for the appearance of the clogging regime. It occurs when $\Delta P_{\text {clogging }} \gtrsim \Delta P_{1}$. Since the bubble density in the shorter arm is about that of close packing, $\Delta P_{1} \simeq c L_{1} / L_{b} \mathrm{Ca}^{2 / 3} \gamma / w$ and the above condition reads

$$
\frac{L_{1}}{L_{b}} \lesssim \frac{\alpha}{c} \frac{L_{b}-w}{w} q^{2 / 3}\left(\mathrm{Ca}^{*}\right)^{1 / 3} \delta .
$$

With $\delta \sim 0.05$ estimated from the amplitude of the backand-forth movement, and for the experimental conditions investigated, the above criterion was evaluated and reads $L_{1} / L_{b} \lesssim 6$. From this analysis, one sees that the loop must be as small as a few bubbles in order to lead to the clogging regime. This is in agreement with the observation that it occurs in the small loop but not in the large one.

This crude approach does not allow us to predict quantitatively the evolution of the regime as a function of $\mathrm{Ca}^{*}$, as the receding amplitude $\delta$ is a priori dependent on the capillary number. It is noteworthy that it experimentally decreases with $\mathrm{Ca}^{*}$, since the droplets deform to go through the junction for $\mathrm{Ca}^{*}$ above $6 \times 10^{-3}$. A detailed modeling of bubble collisions seems necessary to go beyond this crude analysis.

\section{CONCLUSION}

In this work, we have performed a comprehensive study of bubble traffic in microfluidic channels, varying the bubble density from dilute regimes to close packing. We have shown that the bubble transport properties in straight channels are remarkably independent of the density up to close packing, when considering either the bubble relative velocity or the pressure drop due to a single bubble. On the contrary, in the loop geometries investigated, the relative gas flow rate has a strong influence on the bubble traffic. We report striking differences between large and small loops. For loops much longer that the bubble size, several regimes occurs with increasing relative gas flow rate. Similarly to previous 
work, we report and account for a filtering regime at low bubble densities, followed by a repartition regime. Our data unambiguously show that the selection rule at the inlet junction is nontrivial and that the common assumptions that bubbles simply follow the main stream is not valid. We have been able to account quantitatively for the repartition by considering an empiric selection rule at moderate relative gas flow rates and by considering the maximal bubble density at higher rates. In the small-loop situation, we have evidenced a new regime at high gas densities where bubbles are trapped in the long arm of the loop. This situation, similarly to traffic congestion, is due to bubble collisions at the outlet of the loop. These collisions create a back-and-forth displacement of the bubbles that is accompanied by a nontrivial fluid carrier flow in the vicinity of the last bubble, and by a specific drop in pressure that ensures the pressure balance between the two arms. In the large loop, this extra pressure drop is too low compared to the pressure drop between the inlet and the outlet of the loop, explaining why this regime is specific to short loops. We have identified the parameters that are important to tune, i.e., the foam quality, the gas flow rate, and the loop length, to modify droplet traffic behavior significantly. The clogging mechanism that we have evidenced may play an important role in porous media, where the pore structure might be modeled as a succession of small loops. Our experiments suggest that some gas bubbles could be trapped in porous media even in channels of homogeneous size, leading to the formation of preferential paths.

As all the mechanisms do not involve specific issues associated with gas, they are likely to hold with minor modifications for particles whose size is comparable to the channel size. We recall that the criteria for observing clogging is that the pressure induced by the counter-flow balances the drop in pressure of the short arm. We thus believe that the same behavior may be displayed by droplets of immiscible liquids in microchannels or in porous media, for polymer beads or macromolecules in nanochannels.
[1] J. J. Kilbane II, P. Chowdiah, K. Kayser, B. Misra, K. Jackowski, V. Srivastava, G. Sethu, A. Nikolov, D. Wasan, and T. Hayes, Land Contam. Reclam 5, 41 (1997).

[2] S. Wang and C. Mulligan, Water Air Soil Pollut. 157, 315 (2004).

[3] R. F. Li, W. Yan, S. Liu, G. Hirasaki, and C. Miller, SPE J. 15, 928 (2010).

[4] M. J. Fuerstman, P. Garstecki, and G. M. Whitesides, Science 315, 828 (2007).

[5] D. R. Link, S. L. Anna, D. A. Weitz, and H. A. Stone, Phys. Rev. Lett. 92, 054503 (2004).

[6] W. Engl, K. Ohata, P. Guillot, A. Colin, and P. Panizza, Phys. Rev. Lett. 96, 134505 (2006).

[7] D. A. Sessoms, M. Belloul, W. Engl, M. Roche, L. Courbin, and P. Panizza, Phys. Rev. E 80, 016317 (2009).

[8] M. Schindler and A. Ajdari, Phys. Rev. Lett. 100, 044501 (2008).

[9] F. Jousse, R. Farr, D. R. Link, M. J. Fuerstman, and P. Garstecki, Phys. Rev. E 74, 036311 (2006).

[10] M. Belloul, W. Engl, A. Colin, P. Panizza, and A. Ajdari, Phys. Rev. Lett. 102, 194502 (2009).

[11] M. Belloul, L. Courbin, and P. Panizza, Soft Matter 7, 9453 (2011).
[12] D. A. Sessoms, A. Amon, L. Courbin, and P. Panizza, Phys. Rev. Lett. 105, 154501 (2010).

[13] C. N. Baroud, F. Gallaire, and R. Dangla, Lab Chip 10, 2032 (2010).

[14] P. Parthiban and S. A. Khan, Lab Chip 12, 582 (2012).

[15] O. Cybulski and P. Garstecki, Lab Chip 10, 484 (2010).

[16] L. F. Cheow, L. Yobas, and D. L. Kwong, Appl. Phys. Lett. 90, 054107 (2007).

[17] J. Stark and M. Manga, Transp. Porous Media 40, 201 (2000).

[18] S. L. Anna, N. Bontoux, and H. A. Stone, Appl. Phys. Lett. 82, 364 (2003).

[19] M. J. Fuerstman, A. Lai, M. E. Thurlow, S. S. Shevkoplyas, H. A. Stone, and G. M. Whitesides, Lab Chip 7, 1479 (2007).

[20] F. Bretherton, J. Fluid Mech. 10, 166 (1961).

[21] H. Wong, C. Radke, and S. Morris, J. Fluid Mech. 292, 95 (1995).

[22] I. Cantat, Phys. Fluids (1994-present) 25, 031303 (2013).

[23] P. Parthiban and S. A. Khan, Biomicrofluidics 7, 044123 (2013).

[24] L. Du, Ph.D. thesis, University of Bordeaux, 2012.

[25] W. Engl, M. Roche, A. Colin, P. Panizza, and A. Ajdari, Phys. Rev. Lett. 95, 208304 (2005).

[26] This value differs from the constant $\alpha$ introduced in the previous section due to the viscous coupling with the flow in the bubble. 